\title{
Évaluation du risque sur la vie entière dans les analyses de l'UNSCEAR, du BEIR et de la CIPR $^{1}$
}

\author{
Ph. HUBERT*
}

(Manuscrit reçu le 26 septembre 1990)

RÉSUMÉ Ces deux dernières années, les principaux comités internationaux travaillant sur les effets des faibles doses de rayonnement ont publié une nouvelle série d'indicateurs du risque en fonction des doses recues. Ces travaux intègrent les évolutions des données épidémiologiques, notamment depuís la révision de la dosimétrie des personnes exposées à Hiroshima et Nagasaki. Selon les comités, les variantes de calcul - non seulement lors de lanalyse épidémiologique, mais aussi lors du calcul d'un indicateur de risque à la vie entière à partir des "coefficients primaires" fournis par les épidémiologistes - sont extrêmement nombreuses. II s'ensuit que la comparaison et l'interprétation des chiffres annoncés par ces différents comités sont très difficiles. On prẻsente ici une analyse de ces différentes options de calcul et des mesures de leur impact. L'objectif est de bien contrôler les hypothèses de type démographique et les conventions de calcul pour estimer réellement l'effet des hypothèses relevant de l'épidémiologie et de la biologie, jugées ici plus fondamentales. On démontre alors que les résultats sont plus sensibles a ces choix fondamentaux que ne le laissent supposer les données publièes, pour lesquelles beaucoup d'effets se sont apparemment compensés. La mise en place d'une procédure standardisée du calcul du risque à la vie entière (en particulier pour les indicateurs et pour la population de référence) paraît indispensable pour discuter de la portée des hypothèses relatives aux effets biologiques des rayonnements.

ABSTRACT During the last two years, international committees dealing with the effects of ionizing radiation have published a new series of risk estimates. New epidemiological data, in particular in the case of A-bomb survivors whose doses have been reassessed, have been used. Unfortunately, there has been a large range of alternatives, not only in dealing with epidemiological or biological modelling, but also in the actual computation of a life-long risk index which is derived from the epidemiological "primary coefficients". As a consequence, published figures are never truly comparable. The identification of all computational alternatives is made here, and the quantification of their consequences is attempted. The purpose is to achieve the control of demographic assumptions (e.g. reference population) and of various conventional assumptions, in order to measure the impact of epidemiological and biological hypotheses, which are felt to be more fundamental. The analysis shows that such impacts are more important than suggested by published tables. The effects of various alternatives obviously compensate one another. Further discussions on the modelling of the biological effects of radiation would greatly benefit from the development of a "standar" for life-long risk computations.

1. UNSCEAR - United Nations Scientific committee on the effects of atomic radiations. Comité scientifique des Nations Unies sur les effets des rayonnements ionisants.

BEIR - Committee on the Biological effects of ionizing radiations : de l'Académie des sciences des Etats-Unis.

CIPR - Commission internationale de protection radiologique

NRC - National regulatory commission, commission de contrôle des applications de l'énergie nucléaire, Etats Unis.

NIH - National institute of health, Etats Unis.

* Commissariat à l'énergie atomique, Institut de protection et de sûreté nucléaire (IPSN), Département de protection sanitaire, DPS/SEGP, BP 6, 92265 Fontenay-aux-Roses Cedex. 


\section{INTRODUCTION}

La présente analyse vise à mettre en valeur les principales différences dans les modes d'estimation du risque associé aux rayonnements lonisants telles qu'elles apparaissent dans les publications de I'UNSCEAR, du BEIR et de la CIPR $[18,9,1]$. Référence est également faite aux estimations précédentes du NIH [3] et de la NRC [4]. Cette présentation, très simplifiée, se borne à identifier et, éventuellement, à quantifier les alternatives ; elle ne prétend pas juger du bien-fondé de telle ou telle démarche.

La présentation est axée sur l'examen des séquences du calcul ; il s'ensuit que certains points importants, comme l'existence de sous-populations sensibles et le fondement biologique de certains modèles ajustés, ne sont pas mis en valeur (cf. par exemple [5]).

\section{DÉMARCHE SUIVIE DANS L'ESTIMATION D'UN INDICATEUR DU RISQUE}

Pour apprécier les effets dus à une exposition programmée ou accidentelle à des rayonnements ionisants, il faut disposer d'un indicateur qui fournisse une mesure du risque en fonction de la dose. Cet indicateur, souvent appelé indicateur du "détriment" ou du "risque sur la vie entière", ne s'obtient pas simplement à partir des données épidémiologiques. D'autres étapes sont nécessaires dont on fournit ici les principales caractéristiques.

\section{Définition de l'objectif des estimations}

Les estimations du risque sont parfois réalisées pour permettre l'évaluation du niveau de risque et, à partir de là, l'optimisation des pratiques, qu'il s'agisse d'optimisation socio-économique, de stratégies de protection ou de pratiques médicales (cf. justification de dépistages, etc.). L'UNSCEAR et le BEIR sont plutôt rédigés dans cette optique.

Les études peuvent aussi être conçues pour servir de base à un système de radioprotection. Cette optique justifie que l'on ait recours à des "hypothèses prudentes" en situation d'incertitude, mais, en revanche on doit envisager toutes les situations d'irradiation. II est alors fréquent de sacrifier la précision des estimations à l'efficacité du système. La CIPR travaille plutôt dans cette optique, quoique cette approche ne soit plus aussi clairement affirmée qu'auparavant (CIPR 26 [1]).

\section{Description du risque sur la vie entière}

Les effets d'une exposition à une substance cancérogène ou à un rayonnement se traduisent par une modification des paramètres démographiques qui décrivent une population ou un individu lui appartenant (taux de mortalité toutes causes et taux spécifiques pour un ou plusieurs cancers, probabilité de survie à un âge donné, espérance de vie, probabilité de décès à un âge donné). 
La façon la plus simple de visualiser l'excès de risque est de calculer l'excès d'incidence en fonction de l'âge (fig. 1 : ce calcul est fait sur une population japonaise d'individus recevant 0,1 Sv au poumon à 25 et 55 ans, selon les hypothèses de calcul du BEIR ou de L'UNSCEAR). II suffit pour cela de connaître un "coefficient de risque primaire", qui est soit le "risque additif", c'est-à-dire l'excès d'incidence lui-même, soit le "risque relatif", coefficient qu'il faut multiplier par l'incidence naturelle du cancer auquel on s'intéresse.

\section{Décès en excès pour 100000 personnes vivantes à l'âge $X$.}

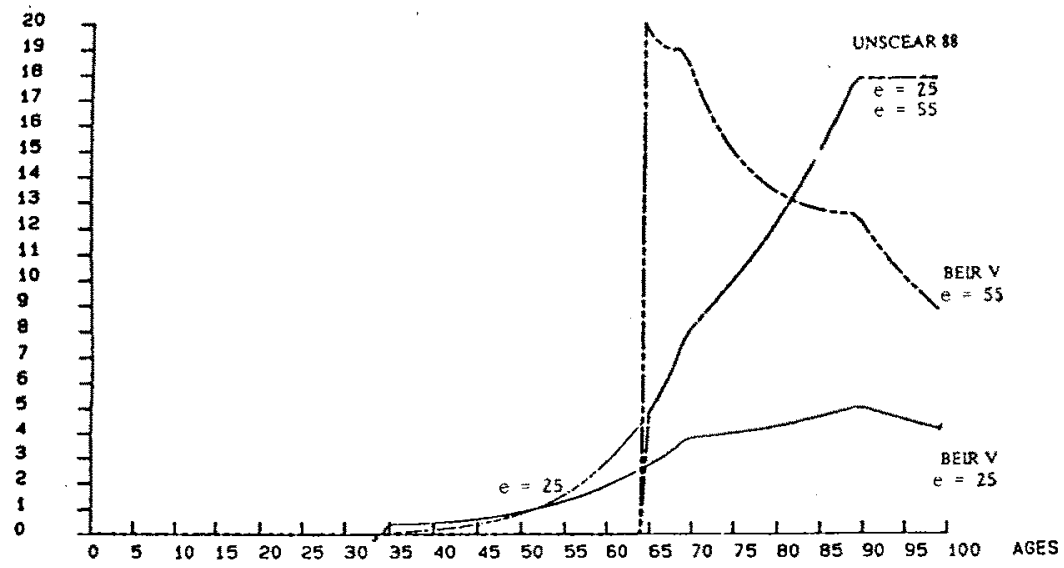

Fig. 1. - Excès d'incidence suite à une irradiation

0,1 Sv au poumon, áge l'irradiation(e) 25 of 55 ans,

coefficlents primaires UNSCEAR 88 et BEIR $\mathrm{V}$ - rieque relatif-population laponalse.

'Décès en excè, pour 100000 personnes vivantes a láge a l'irradiation.

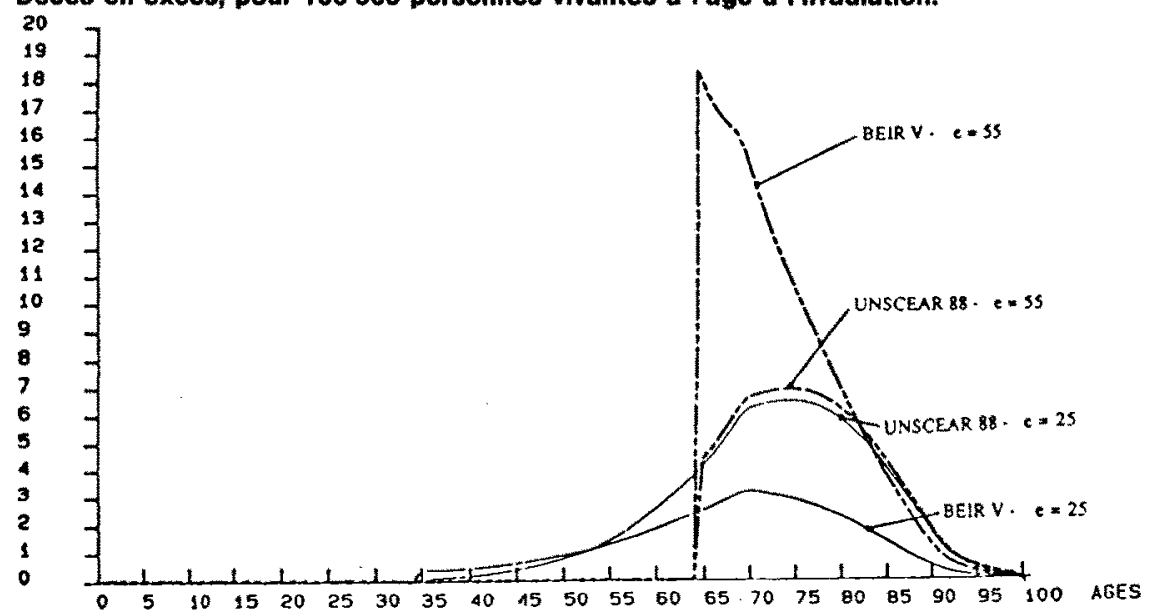

Fig. 2. - Probabilités de décéder à un âge exact suite à une irradiation pour : 0,1 SV au poumon, Ege à l'irradiation (e) 25 et 55 ans,

coefficients primaires UNSCEAR 88 et BEIR $V$ - risque relatif-population japonaise. 
De là, connaissant la courbe de survie des individus, on en déduit les probabilités supplémentaires de décéder aux divers âges (fig. 2), produits de l'excès d'incidence (fig. 1) par la probabilité d'avoir survécu jusqu'à cet âge.

La totalité de l'information sur l'excès de risque est contenue dans ces courbes, mais les graphiques sont difficiles à manipuler et peu parlants. Des indicateurs synthétiques sont donc utilisés ; on parle à leur sujet d'indicateurs du détriment. Le plus courant est l'excès de cancer sur la vie entière. Deux notions légèrement différentes sont utilisées par I'UNSCEAR 88, et par le BEIR V. L'indicateur le plus intuitif, l'ERL ${ }^{2}$ du BEIR est la différence entre le nombre de cancers qui serait observé dans une cohorte irradiée et celuí observé dans une cohorte non irradiée. Le concept correspondant à l'ELR est proche du concept de risque attribuable; mais l'indicateur peut devenir négatif pour un cancer donné à cause de la "compétition" entre cancers. En effet, l'accroissement global de mortalité peut faire que l'on observe moins de cancers sur une localisation précise. L'autre indicateur, utilisé par l'UNSCEAR et la CIPR, noté LREID ${ }^{3}$, est plus difficile à interpréter, mais plus stable (il est toujours positif ${ }^{4}$. Formellement, c'est l'intégrale de la courbe des probabilités supplémentaires (fig. 2). II représente le nombre des cas qui seraient dus à l'irradiation, au sein d'une cohorte irradiée (nombre qui serait compté sil les cancers radioinduits étaient identifiables).

Les différents comités calculent aussi la perte d'espérance de vie. Cet indicateur n'est pas toujours mis au premier plan, mais il est de plus en plus utilisé. Comme concept du détriment il est aussi intéressant, sinon plus, que le surcroît de mortalité, l' excès" de cancer n'étant qu'une mortalité anticipée dont il est utile de savoir si elle se produit aux âges jeunes ou avancés. Un avantage non négligeable de la perte d'espérance de vie en tant qu'indicateur est qu'elle est peu sensible à l'alternative entre la projection du risque absolu et celle du risque relatif. Les données publiées sont malheureusement trop limitées pour permettre une analyse équivalente à celle faite ici sur les indicateurs de mortalité.

Les indicateurs précédents ne résument pas toute l'information des courbes, mais ils se suffisent à eux-mêmes; d'autres ont été proposés qui ne peuvent être utilisés qu'en complément, par l'exemple, l'espérance de vie

2. ELR. Excess lifetime risk : excès de risque sur la vie entière.

3. LREID. Lifetime risk of exposure induced deaths : risque sur la vie entière de décès induits par l'exposition.

4. Pour mieux comprendre la différence entre deux indicateurs, on peut examiner l'exemple suivant. On considère deux cohortes de 100000 personnes. Pour la cohorte non irradiée, on observe 300 cancers du poumon, et pour la cohorte irradiée, on en observe 330 . Le risque attribuable, ou l'ELR, sera donc de 30 cas. Si l'on détaille ce qui se passe dans la seule cohorte irradiée, les cancers "radioinduits" auront accru la mortalité, de sorte que les cancers "naturels" n'auront pas tous pu s'exprimer et il y aura, par exemple, 298 cancers "naturels" (en tout cas, moins de 300 ) et 32 cancers radioinduits. Le LREID sera dono de 32 . En effet, chaque fois que l'on augmente les taux de décès en ajoutant une cause ou en augmentant une déjà existante, tous les autres nombres attendus de décès pour les autres causes régressent (la probabilité totale de décès est, en effet, immuablement fixée à 1).

De même, si l'on irradie deux organes, dont l'un est très exposé ou très radiosensible, on observera un excès d'incidence pour les deux, mais les nombreux décès dus à lirradiation de l'organe sensible réduiront la base sur laquelle l'autre risque peut s'exprimer au point d'annuler, parfois complètement, l'effet de l'augmentation de l'incidence. C'est ainsi que des calculs peuvent aboutir à un ELR négatif. 
perdue par cancer radio-induit, ou l'âge moyen au décès. La CIPR, dans son projet de recommandation, est seule à publier une liste fournie d'indicateurs; il n'y a donc pas de comparaison possible avec le BEIR ou I'UNSCEAR sur ces points.

\section{L'enchaînement des calculs et les principales alternatives}

Le calcul d'un indicateur du risque à la vie entière implique deux étapes de calcul presque totalement disjointes ; d'abord l'estimation des "coefficients de risque primaire", puis celle du risque sur la vie entière. S'y associent de nombreuses hypothèses, internes à chaque étape, relatives au passage d'une étape à l'autre et, enfin, à la validité des résultats globaux dans leur application à d'autres situations.

Etape 1 : Estimation de "coefficients primaires" de risque sur des populations irradiées. II s'agit du travail épidémiologique à proprement parler. Les décisions à prendre sont assez bien connues : choix de la population (Hiroshima et Nagasaki, spondylarthrite, fluoroscopies et autres irradiations thérapeutiques ou diagnostiques, mélange de populations), choix d'une courbe à ajuster dérivée soit d'un modèle statistique (linéaire, probit, logit, Weibull) soit d'un modèle biologique (linéaire, linéoquadratique, multi-étape), ensemble de choix classiques dans une étude épidémiologique (groupe témoin, constitution des classes, etc.).

Comme dans toute étude de ce type, on veut estimer une relation entre la dose et le risque absolu (nombre de cas supplémentaires par an et par unité de dose) ou entre la dose et le risque relatif (fraction supplémentaire de l'incidence naturelle par unité de dose). Le BEIR V, la CIPR et, moins nettement, I'UNSCEAR, ont utilisé le risque relatif, parce que c'est ce coefficient qu'ils "projettent" au-delà de la durée d'observation de la cohorte. Pour désigner ce modèle, on parle de risque multiplicatif, par opposition au modèle du "risque additif" dans lequel c'est le risque absolu qui est projeté.

L'excès de risque, relatif ou absolu, peut varier avec l'âge l'irradiation, l'âge à l'observation (c'est-à-dire au décès) et le sexe. Cependant, cette variation est difficile à mesurer, c'est pourquoi les avis diffèrent sur les méthodes d'estimation, certains jugeant même plus raisonnable de ne pas chercher à quantifier ces effets (cf. UNSCEAR 88 où l'excès du risque ne varie qu'avec la dose).

II faut noter, à ce propos, que la CIPR et l'UNSCEAR n'ont pas calculé eux-mêmes de coefficients primaires, ils ont utilisé les résultats de la RERF $[11]^{5}$ pour Hiroshima et Nagasaki. En revanche, le calcul des coefficients primaires a été effectué directement par le BEIR.

Étape 2 : Estimation du risque sur la vie entière à partir des coefficients de risque primaire. II s'agit là de calculs démographiques (cf. fig. 1 et 2). Ces calculs ont été effectués dans le cadre des travaux du BEIR et

5. Radiation effect research foundation (Fondation pour la recherche sur les effets des radiations), organisme chargé des investigations épidémiologiques sur Hiroshima et Nagasaki. 
de I'UNSCEAR (code CEPN-INSERM [8]) et de la CIPR (Annexe C de B. Lindell). Les types de choix faits sont, en général, peu commentés ; certains relèvent de l'hypothèse de calcul, d'autres de la simple convention.

Parmi les choix qui relèvent de la convention, il faut citer celui du ou des indicateurs de détriment, et celui de la "population de référence" c'est-à-dire des données suivantes: taux de mortalité générale, taux de mortalité spécifique pour des cancers particuliers, structure d'âge. Généralement, la "population de référence" est simplement définie par les paramètres démographiques d'un pays particulier (Japon, Etats Unis, etc.); ce choix est important car les taux varient beaucoup.

Les tables démographiques traditionnelles fournissent des données "transversales" (c'est-à-dire observées dans l'année) ; elles sont aussi appelées tables "de l'année" ou du "moment". II s'ensuit que, dans la table, les taux de décès "0-1 an" sont ceux d'enfants nés dans l'année, tandis que ceux "80-81 ans" s'appliquent à des gens nés il y a quatrevingts ans. En théorie, ces données sont donc inadaptées pour décrire une cohorte. Tous les comités ont, cependant, retenu l'approche transversale, ses défauts étant compensés par une longue pratique. D'autres particularités des divers codes tiennent à la façon de reconstituer les taux de décès, surtout s'ils sont spécifiques de certains cancers, après 80 ans environ. En effet, le flou statistique et l'ancienneté des générations rendent difficilement utilisables les données observées.

Liaison entre les étapes 1 et 2 : le problème du "transport". Des hypothèses sont nécessaires pour appliquer les coefficients de risque primaire sur la population de référence. Elles ne sont généralement pas discutées. Si l'on utilise le risque relatif (ou le risque absolu) calculé sur une population pour l'appliquer à une autre, cela signifie qu'on considère que l'on peut le projeter non seulement au-delà de la période d'observation, mais aussi d'une population à une autre. Cette dernière opération est qualifiée de "transport du coefficient primaire". II est envisageable de considérer que c'est le risque relatif qui se projette après la période d'observation mais que c'est le risque absolu qui se transporte. C'est ce qu'avait fait le NIH [3].

Le BEIR a transporté le risque relatif, ainsi que l'UNSCEAR 88 ; en effet, le fait que ce dernier comité ait travaillé sur la population japonaise n'empêche pas qu'il y ait eu transport, car la population d'Hiroshima et de Nagasaki en 1945 n'est pas représentative a priori de celle du Japon en 1982. Ces hypothèses n'ont pas été commentées dans les documents.

Dans l'annexe $\mathrm{C}$ du projet de recommandations de la CIPR, c'est le risque relatif qui est transporté ; dans l'annexe $B$, les auteurs ont effectué la moyenne entre les résultats obtenus par le transport du risque 
relatif et ceux obtenus par le transport du risque absolu. C'est de cette moyenne que sont issus les facteurs de pondération des organes.

Validité de l'application à des sítuations de radioprotection. Lorsqu'il est indispensable de faire des choix pour mener à terme les calculs, ceux-ci sont bien décrits. En revanche, s'il n'est pas besoin de formuler une hypothèse pour aboutir à un résultat chiffré, celle-ci reste trop souvent implicite, alors qu'il est déraisonnable d'utiliser les résultats sans y faire référence.

Beaucoup de présupposés sont ainsi associés aux indicateurs du risque : absence de sous-populations spécialement sensibles à la radiocancérogenèse, absence de synergie avec les autres cofacteurs (à noter que la synergie est définie différemment selon que l'on utilise le risque absolu ou le risque relatif), etc.

Une attention particulière doit être accordée à l'effet de la distribution temporelle de la dose (débit, fractionnement, étalement). Cette distribution dans les cohortes irradiées étudiées (fort débit à Hiroshima et Nagasaki, fortes doses et fractionnement pour les irradiations médicales) est généralement différente de celle que l'on considère pour l'exposition des travailleurs, du public ou de patients en radiodiagnostic. Comme il n'est pas nécessaire de prendre position pour mener à terme le calcul, ce point est abordé séparément, généralement en commentaire. Les comités proposent de le traiter par un facteur multiplicatif à appliquer au résultat final. Seule la CIPR a proposé une valeur $(0,5)$, étayée par des commentaires assez brefs. Les autres comités ont fait des suggestions (UNSCEAR 88 : de 0,5 à 0,1 ; BEIR $V: 0,5$ pour la leucémie). Antẻrieurement, le NIH avait utilisé 0,4 et la NRC 0,2 . La dernière synthèse importante sur la question semble être celle du NCRP ${ }^{6}$ quil remonte à 10 ans [10]

\section{APPROCHES RETENUES ET VALEURS PROPOSÉES}

\section{Rappel des principales hypothèses (Tableau I)}

Le tableau I résume les principales options de calcul des trois comités. On y retrouve les questions discutées ci-avant, mais il s'y ajoute de nombreux choix plus empiriques (par exemple, regroupement des cancers, etc.).

6. National council on radiation protection and measurements, Etats Unis. 
Ph. HUBERT

TABLEAU I

Principales différences dans les estimations du BEIR $V$, de I'UNSCEAR 88 et de la CIPR

\begin{tabular}{|c|c|c|c|c|}
\hline Choix et hypothèses & BEIR V & UNSCEAR 88 & CIPR (Annexe B) & CIPR (Annexe C) \\
\hline $\begin{array}{l}\text { Indicateur du risque } \\
\text { à la vie entière }\end{array}$ & ELR & LREID & LREID & LREID \\
\hline $\begin{array}{l}\text { Population de réfé- } \\
\text { rence : taux }\end{array}$ & USA & Japon & $\begin{array}{l}\text { Mélange de } \\
\text { pays }\end{array}$ & $\begin{array}{l}\text { Japon (+ } \\
\text { Suède) }\end{array}$ \\
\hline $\begin{array}{l}\text { Population de réfé- } \\
\text { rence : structure }\end{array}$ & Stationnaire & "du moment" & $?$ & $?$ \\
\hline $\begin{array}{l}\text { Ajustement des taux } \\
\text { de cancer }\end{array}$ & Données brutes & Données brutes & $?$ & $\begin{array}{l}\text { Fonction expo- } \\
\text { nentielle }\end{array}$ \\
\hline $\begin{array}{l}\text { Dernier groupe d'âge } \\
\text { pour les taux de } \\
\text { cancer }\end{array}$ & $>85$ ans & $>75$ ans & $?$ & $?$ \\
\hline $\begin{array}{l}\text { Estimation dans le } \\
\text { dernier groupe, taux } \\
\text { spécifiques }\end{array}$ & Constant & $\begin{array}{l}\text { Tendanciel } \rightarrow 90 \\
\text { ans, constant } \\
\text { ensuite }\end{array}$ & $?$ & $?$ \\
\hline $\begin{array}{l}\text { Estimation dans le } \\
\text { dernier groupe : taux } \\
\text { toutes causes }\end{array}$ & $\begin{array}{l}\text { Tableau détaillé } \\
\text { (année par } \\
\text { année) }\end{array}$ & cf. supra & & \\
\hline $\begin{array}{l}\text { Dépendance du } \\
\text { risque relatif }\end{array}$ & $\begin{array}{l}\text { Âge à lirradia" } \\
\text { tion, âge à } \\
\text { l'observation, } \\
\text { fonction logis" } \\
\text { tique }\end{array}$ & Constant & $\begin{array}{l}\text { Âge à l'irradia" } \\
\text { tion, ajuste- } \\
\text { ments séparés }\end{array}$ & $\begin{array}{l}\text { Âge à l'irradia- } \\
\text { tion, ajuste- } \\
\text { ments séparés }\end{array}$ \\
\hline $\begin{array}{l}\text { Transport du coeffi- } \\
\text { cient primaire }\end{array}$ & Risque relatif & Risque relatif & $\begin{array}{l}\text { Risque absolu } \\
\text { et risque relatif } \\
\text { (mélange) }\end{array}$ & Risque relatif \\
\hline $\begin{array}{l}\text { Données épidémiolo- } \\
\text { giques }\end{array}$ & $\begin{array}{l}\mathrm{H} \text { et } \mathrm{N}+\text { autres } \\
\text { selon site }\end{array}$ & $\mathrm{H}$ et $\mathrm{N}$ & $\mathrm{H}$ et $\mathrm{N}$ & $\mathrm{H}$ et $\mathrm{N}$ \\
\hline $\begin{array}{l}\text { Groupement de } \\
\text { cancers }\end{array}$ & $\begin{array}{l}\text { Respiratoire } \\
\text { digestif }\end{array}$ & $\begin{array}{l}\text { Pulmonaire } \\
\text { œsophage } \\
\text { estomac, colon }\end{array}$ & & \\
\hline $\begin{array}{l}\text { Effet du débit } \\
\text { de dose }\end{array}$ & $\begin{array}{l}\text { Pas de propo- } \\
\text { sition }\end{array}$ & $\begin{array}{l}\text { Pas de propo- } \\
\text { sition }\end{array}$ & facteur 2 & facteur 2 \\
\hline
\end{tabular}

ELR : Excess lifetime risk : excès de risque sur la vie entière.

LREID : Lifetime risk of exposure induced death : risque sur la vie entière de cancers radioinduits.

H : Hiroshima.

N : Nagasaki. 
Pour décrire plus précisément les différences entre ces approches, il faut distinguer l'estimation du risque "site par site" et celle qui s'effectue "tous cancers confondus". Dans le second cas (tous cancers), on note les points suivants :

\section{Approche de I'UNSCEAR :}

- utilisation d'Hiroshima et Nagasaki,

- ajustement d'un risque relatif moyen (sexe, âge...),

- vérification de la cohérence avec les autres données épidémiologiques,

- calcul du risque à la vie entière sur la population japonaise,

- transport du risque relatif,

- utilisation du code CEPN-INSERM,

- commentaire sur l'effet du débit de dose.

\section{Approche du BEIR :}

- utilisation d'Hiroshima et Nagasaki et des données sur les spondylarthrites [12],

- ajustement d'un risque relatif variant avec le sexe, l'âge à l'irradiation et celui à l'observation (relation fonctionnelle ajustée par régression logistique),

- transport du risque relatif,

- calcul du risque à la vie entière sur la population des Etats Unis,

- utilisation du code de calcul du BEIR,

- commentaire sur l'effet du débit de dose,

- estimation de l'incertitude par simulation.

Approche de la CIPR (Annexes C et B) :

- utilisation d'Hiroshima et Nagasaki,

- ajustement d'un risque relatif variant avec le sexe et l'âge à l'irradiation (ajustements séparés selon les tranches d'âge),

- calcul du risque à la vie entière sur la population du Japon,

- dans l'annexe $\mathrm{C}$, moyenne entre les transports du risque absolu et du risque relatif,

- utilisation du code CIPR (B. LINDELL),

- proposition sur l'effet du débit de dose.

On remarque alors que les données épidémiologiques ne sont pas exactement les mêmes, mais l'introduction des spondylarthrites influe peu (cf. [1, annexe f]). Si le risque relatif est systématiquement utilisé, il peut être soit constant, soit variable avec les âges à l'exposition et à l'observation. Les populations de référence sont différentes (Japon et USA), ainsi que les codes servant au calcul du risque à la vie entière et, enfin, le problème du "transport" n'est pas traité de façon homogène. 
Si l'on examine le calcul de taux de cancers spécifiques, il faut ajouter les précisions suivantes:

Approche de I'UNSCEAR :

- inchangée.

Approche du BEIR V :

- les données de base sont plus nombreuses [12] ; pour le sein, sont ajoutées les études sur les fluoroscopies au Canada et dans le Massachusetts ainsi que sur le traitement des mastites post-partum, pour la thyroïde, les études sur le traitement de la teigne et de l'hypertrophie du thymus chez les enfants ont aussi été utilisées ; pour le reste, l'approche est la même.

Approche de la CIPR :

- dans l'annexe $C$, les risques n'ont pas été estimés pour des cancers spécifiques ; dans l'annexe $B$, les risques sont estimés pour chaque type de cancer en suivant une démarche assez complexe ; les contributions des différents cancers au risque total ont été calculées pour plusieurs pays et avec les deux modèles de transport ; elles ont ensuite été moyennées ; les risques par organe ont été déduits du risque global à partir des contributions moyennes.

Les estimations de coefficients propres à la mesure du risque d'un cancer donné font donc intervenir des choix plus nombreux, notamment parce que l'éventail des données épidémiologiques utilisables s'élargit.

\section{Les estimations proposées}

Après avoir décrit les différents cheminements, il est intéressant d'examiner la variabilité des coefficients finalement proposés. Pour l'exposition d'une population générale, les trois comités ont publié des coefficients pour l'excès de décès par cancer dû à une irradiation au corps entier de 0,1 Sv. Ces résultats sont fournis sans facteur d'atténuation pour le débit de dose (tableau II).

Indéniablement, les propositions qui sont faites pour les indicateurs sont très proches. S'agissant de populations générales (par opposition à des groupes d'âge précis) et assez proches (Japon et Etats Unis sont deux pays à forte espérance de vie et à populations plutôt vieilles), et d'un calcul tous cancers confondus, la proximité peut s'expliquer ; on raisonne sur des moyennes qui peuvent gommer l'effet de l'utilisation de stratégies différentes. Dans le cas de coefficients spécifiques pour certains cancers, le faible écart entre les propositions est plus surprenant. II est peu plausible que l'effet cumulé des multiples choix évoqués ci-avant soit aussi limité, il est plus probable que les divers écarts se sont compensés dans les configurations particulières de ces calculs. Pour examiner cette question, il est donc nécessaire de reprendre étape par étape les calculs aboutissant à une estimation du risque à la vie entière. C'est ce qui est fait ci-après, d'abord pour les hypothèses du calcul du risque à la vie entière, puis sur celles de l'estimation des coefficients primaires. 
TABLEAU ॥

Estimation du risque à la vie entière

Excès de cancers $\left(10^{-5} \mathrm{~Sv}^{-1}\right)$

\begin{tabular}{|l|c|c|c|}
\hline \multicolumn{1}{|c|}{ Cancer ou site irradié } & CIPR Annexe B & UNSCEAR 88 & BEIR V \\
\hline Leucémie & 90 & 97 & 95 \\
Cancer des os & 10 & & \\
Tous cancers sauf leucémie et os & 900 & 610 & 690 \\
Myélome multiple & - & 22 & - \\
Poumon & 180 & 151 & $170^{(1)}$ \\
Thyroïde & 15 & - & \\
Sein (2) & 100 & 60 & 70 \\
Ovaires & 190 & 31 & - \\
Colon & 70 & 79 & \\
Oesophage & 220 & 34 & $240^{(3)}$ \\
Estomac & 40 & 126 & - \\
Foie & 40 & - & - \\
Vessie & 4 & 39 & 260 \\
Peau & 91 & 118 & 785 \\
Autres organes & 1000 & 712 & - \\
\hline
\end{tabular}

(1) appareil respiratoire

(2) taux pour le sexe féminin (à diviser par 2 pour le calcul du risque tous organes sur la population générale)

(3) système digestif

Les deux estimations ainsi comparées sont celles du BEIR et de I'UNSCEAR, pour lesquelles tous les détails ont pu être recueillis, et dont tous les résultats définitifs ont été publiés.

\section{IMPACTS DES OPTIONS DE CALCUL}

\subsection{Calcul du risque sur la vie entière}

Dans le calcul du risque à la vie entière, plusieurs éléments interviennent. On présente ici une comparaison terme à terme, "toutes choses égales par ailleurs". Ces travaux ont été réalisés en collaboration avec un groupe de chercheurs ayant travaillé pour chacun des trois comités $[13,9,1]$ dans le cadre d'un séminaire organisé par le Centre d'étude pour l'évaluation de la protection dans le domaine nucléaire (CEPN) au Centre d'études nucléaires de Fontenay-aux-Roses avec le soutien de la DG XI de la Commission des Communautés européennes. 


\section{Comparaison des codes de calcul sur la vie entière}

Un travail d'intercomparaison a été mené entre le code CEPN-INSERM et celui du BEIR. Ces deux codes de type démographique (cf. I) ont été appliqués aux coefficients de risque primaire de l'UNSCEAR 88 et à la population japonaise. Les écarts, testés sur trois cancers (leucémie, sein, poumon) et trois âges, sont restés limités : 5 à $10 \%$ environ sur l'excès de mortalité par cancer, un peu plus pour la perte d'espérance de vie (jusqu'à $20 \%$ pour une exposition du poumon à 50 ans). Eu égard aux incertitudes sur l'estimation des coefficients primaires ou sur les règles d'extrapolation, ces chiffres sont satisfaisants. Toutefois, on peut les considérer comme regrettables parce qu'ils introduisent, sans raison particulière, un flou quand on compare des techniques d'ajustement.

De surcroît, une analyse plus détaillée a montré que ces écarts sont restés limités à cause d'effets qui se compensent. Par exemple, si l'on regarde l'excès de mortalité exprimé avant 65 ans pour une irradiation au poumon à 0 an, l'écart est de $20 \%$ au lieu de $10 \%$ sur toute la durée de vie. Les deux codes ne calculent pas exactement le même indicateur d'excès de mortalité (ELR dans un cas, LREID dans l'autre) ; l'écart, mesuré sur l'ensemble des cancers, atteint $15 \%$, mais il ne dépasse pas $3 \%$ pour une irradiation partielle. De même, le code du BEIR garde le taux constant à l'intérieur d'une tranche d'âge, tandis que celui du CEPNINSERM interpole et extrapole. Si les tranches d'âge sont de 10 ans, l'écart peut atteindre $5 \%$ sur le cancer du poumon. Les effets les plus importants s'observent pour l'irradiation de personnes assez âgées. Ceci n'est pas trop préoccupant dans la mesure où le coefficient de risque primaire est, lui, beaucoup mieux connu pour les âges avancés, ce qui compense l'imprécision démographique.

Ces deux codes sont donc assez proches et, lorsque l'on compare des résultats globaux (tableau II), on peut affirmer qu'ils n'introduisent pas de différences majeures. L'exercice d'intercomparaison n'a pu être mené avec le code de la CIPR, annexe C. II semble peu probable que la différence méthodologique (les taux de mortalités spécifiques ne sont pas pris directement dans les statistiques pour chaque tranche d'âge mais déduits d'une fonction ajustée) conduise à une importante source d'écarts quantitatifs.

\section{Autres alternatives de calcul}

Mention a déjà été faite du choix du concept de détriment (LREID ou ELR), dont on a vu que l'enjeu est plus méthodologique que quantitatif, sauf si l'on fait entrer en compétition les effets de l'irradiation simultanée de deux organes. En revanche, il faut bien mesurer les enjeux de l'utilisation de données transversales, commune aux trois comités. En premier lieu, ces données évoluent, parfois assez vite. Au rythme actuel, l'espérance de vie en France augmente de plus d'un an tous les quatre ans, car les taux de mortalité généraux baissent régulièrement. Si l'on cherchait à suivre la cohorte des individus nés cette année, on observerait une espérance de vie et, donc, un excès de mortalité par cancer radio-induit supérieurs à ceux calculés avec les tables du "moment" de leur 
naissance. La récente querelle sur la "descendance finale des générations" illustre bien les difficultés associées à la manipulation des données transversales.

Le problème principal tient, toutefois, à l'établissement de taux pour des cancers spécifiques. Par exemple, le cancer du poumon voit aujourd'hui son incidence augmenter fortement (fig. 3). Le recours à des données transversales sous-estime donc le risque par rapport à un calcul basé sur une incidence naturelle correspondant au suivi de générations. L'évolution du taux avec l'âge est aussi très trompeuse ; ici l'"effet génération" laisse croire à une baisse de l'incidence avec l'âge, alors que le suivi d'une génération montre une croissance constante.

Taux de deces (pour 100 000)

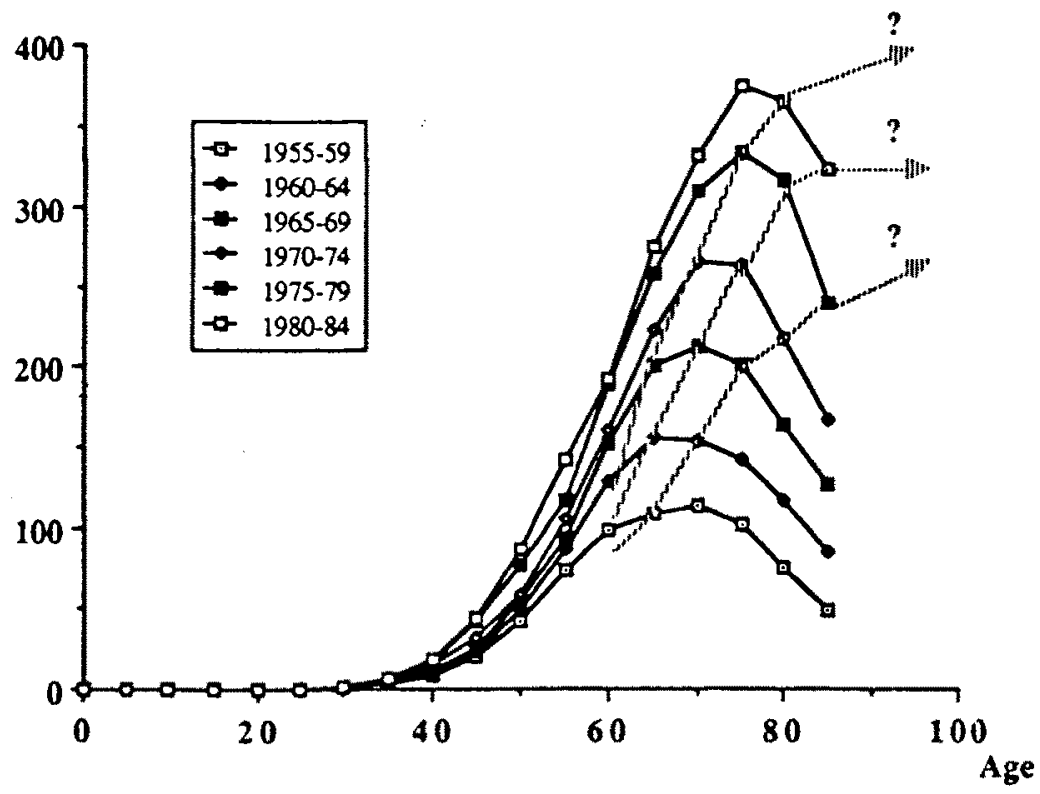

Fig. 3. - Analyses transveraales et longitudinales du cancer du poumon. Taux de mortalité par cancer du poumon aux différents áges : les pieges de l'analyse transversale (FRANCE) [6].

analyses transversales,

analyses longltudinales

Lorsque l'on considère un cancer comme celui du poumon, si l'on veut effectuer un calcul longitudinal, des facteurs de l'ordre de 2 voire 3 sont associés à la façon de modéliser l'évolution de l'“incidence naturelle" des cancers. Aujourd'hui, le recours aux données transversales évite d'avoir à formuler ces hypothèses, mais il sera, sans doute, bientôt nécessaire d'ouvrir le débat. 


\section{Les populations de référence}

Le choix de la population de référence influe sur trois paramètres : la survie globale des cohortes, puis, si l'on fait un calcul pour l'irradiation "d'une population", la structure d'âge et, enfin, les "incidences naturelles" de cancers.

Les effets de la structure d'âge ne sont pas négligeables. Ori peut les estimer en comparant, dans un même pays, la population "stationnaire" (pyramide des âges fictive obtenue en supposant le remplacement des générations) et la population réelle. Ils atteignent $10 \%$ aux Etats-Unis. Ils sont maximaux si l'on compare un "pays jeune" et un "pays vieux". L'UNSCEAR a comparé le Royaume Uni et Puerto Rico, pays où l'espérance de vie est similaire (73,7 contre 73,9 ans). L'excès de risque à Puerto Rico, avec le modèle additif, est supérieur de $25 \%$ pour la mortalité et de $50 \%$ pour l'espérance de vie. Cet exemple reste, toutefois, exceptionnel et les effets de ce type sont peu importants.

En ce qui concerne l'incidence naturelle des cancers, l'effet "tous cancers confondus" est généralement limité. En revanche, pour des cancers spécifiques, l'utilisation d'un coefficient primaire de risque relatif va aboutir à des écarts d'un facteur 4 , comme le prouve l'écart entre les incidences naturelles mesurées par les taux "standardisés" de l'OMS (tableau III).

\section{TABLEAU II}

Taux de décès pour certains cancers dans différents pays.

Taux annuels (pour 100000 ) standardisés pour la population "européenne" [7]

\begin{tabular}{|lc|c|c|c|}
\hline & & $\begin{array}{c}\text { Poumon } \\
(H+F)\end{array}$ & $\begin{array}{c}\text { Sein } \\
(F)\end{array}$ & $\begin{array}{c}\text { Estomac } \\
(H+F)\end{array}$ \\
\cline { 3 - 5 } Etats-Unis & 1984 & 53 & 32 & 6 \\
Japon & 1984 & 25 & 8 & 41 \\
G.B. & 1985 & 57 & 42 & 16 \\
France & 1986 & 32 & 27 & 10 \\
Argentine & 1982 & 30 & 27 & 15 \\
\hline
\end{tabular}

\section{Bilan des variantes de calcul}

A cette étape, les effets associés aux types de traitements effectués par les codes (type de lissage, etc., indicateurs exacts) sont assez minimes. Ceux qui relèvent du choix d'une population de référence, quoique non négligeables, restent limités tant que l'on travaille sur une population générale sans spécifier le site irradié. 
Mais en ce qui concerne la radio-induction de cancer sur un organe, les conséquences de ce choix conventionnel sont considérables quand on transporte le risque relatif. Si les coefficients primaires de risque étaient les mêmes dans les analyses de l'UNSCEAR et du BEIR $V$, les écarts devraient être de 400 ou $500 \%$ (cf. tableau III) sur les cancers du sein et de l'estomac, et non de 15 à $20 \%$ (tableau II).

\subsection{Effet des techniques d'estimation du coefficient de risque primaire}

Après avoir estimé les impacts associés à la mise en œuvre du calcul de risque sur la vie entière, il convient d'examiner les enjeux des décisions relatives au traitement des données épidémiologiques. Aujourd'hui, les techniques d'estimation des coefficients primaires diffèrent essentiellement sur la facon de traiter la variation temporelle de l'excès du risque (absolu ou relatif) qui peut varier tant avec l'âge à l'irradiation qu'avec l'âge à l'observation. L'UNSCEAR 88 l'a supposé constant dans le calcul qu'il propose comme final, mais il a effectué aussi des calculs en supposant qu'il varie avec le sexe et l'âge à l'irradiation. Ces derniers ont été repris par la CIPR dans certains calculs spécifiques (annexe C). Les coefficients de risque primaire associés à cette approche sont, en fait, disponibles dans le rapport RERF [11]. Le BEIR $V$ a suivi une approche différente en admettant que le risque relatif variait avec tous ces paramètres. II a, de plus, supposé que cette variation était une fonction explicite de ces paramètres, ajustée sur l'ensemble des données, alors que le RERF et donc I'UNSCEAR estiment les coefficients séparément dans chaque "strate" (cf. annexe).

Les coefficients primaires de risque ainsi obtenus diffèrent, mais cette différence n'a pas tant d'intérêt en soi ; pour juger de son enjeu, il faut examiner l'impact sur l'indicateur du risque à la vie entière. Ceci doit être fait "toutes choses égales par ailleurs" et il faut contrôler la population de référence et le code. Ceci a été fait en reprenant le code CEPNINSERM et les données japonaises, et en l'appliquant à deux cancers (sein et poumon). Le cancer du sein est intéressant parce que le BEIR a ajusté ses données sur d'autres données que celles d'Hiroshima et de Nagasaki, et le cancer du poumon l'est parce que, au contraire, c'est uniquement le modèle ajusté qui change. Les calculs ont été faits pour 25 et 55 ans.

Au départ, la différence entre les coefficients de risque primaire pour le poumon est très grande (fig. 4). Les résultats intermédiaires du calcul à la vie entière (fig. 1 et 2) montrent que les différences s'atténuent. En effet, les gros écarts observés aux âges relativement jeunes sur le coefficient de risque relatif s'appliquent à des incidences naturelles très faibles. Finalement, les écarts observés sur le coefficient primaire ne sont pas répercutés entièrement sur le risque à la vie entière. L'essentiel des décès par cancer du poumon se produit entre 65 et 85 ans et de gros écarts sur le coefficient primaire en deçà et au-delà de cet âge sont de peu d'importance. 
Risque relatif

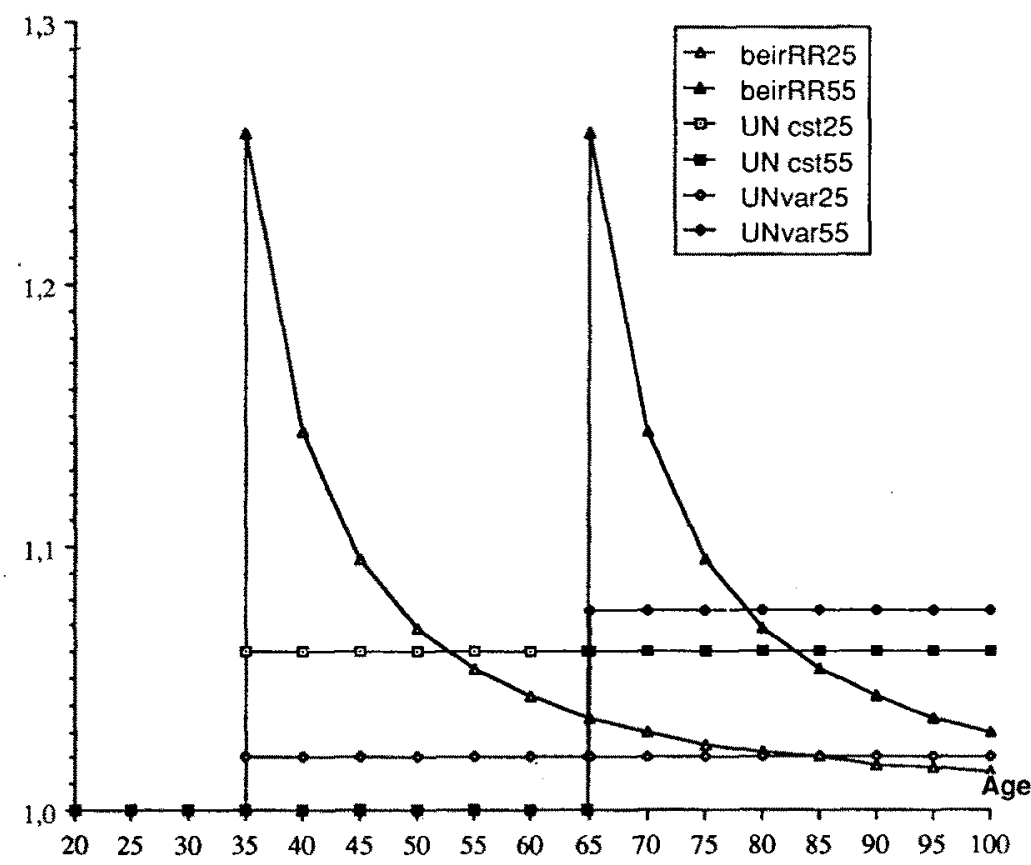

Fig. 4. - Coefficient de risque relatif

pour 0,1 Sv au poumon, age lirradiation 25 et 55 ans, coefflcients primalres UNSCEAR 88 et BEIR $V$ - risque reletif.

\footnotetext{
beir RR25 : BEIR, risque relatif calculé pour une exposition à 25 ans

beir RR55 : BEIR, risque relatif calculé pour une exposition à 55 ans

UN cst25 : UNSCEAR 88, risque relatif constant, pour une exposition à 25 ans

UN cst55 : UNSCEAR 88 , risque relatif constant, pour une exposition à 55 ans

UN var25 : UNSCEAR 88, risque relatif variable avec l'âge à l'exposition, pour une exposition à 25 ans

UN var55 : UNSCEAR 88 , risque relatif variable avec l'âge à l'exposition, pour une exposition à 55 ans
}

II reste, cependant, pour une irradiation à un âge donné, des écarts importants (tableau IV). Pour le cancer du poumon malgré les facteurs 2 entre le modèle en risque relatif constant et le modèle du BEIR, chez les jeunes et les quinquagénaires, on comprend que, sur la "population entière", les résultats soient proches, puisqu'll y a compensation entre les deux effets chez ces deux cohortes. On note aussi que le calcul fondé sur un coefficient de risque relatif (noté risque relatif variable UNSCEAR), variable avec l'âge à l'exposition, mais invariant avec l'âge à l'observation, aboutit à des résultats proches de ceux du BEIR (cf. tabl. IV). Cette dernière optique a été retenue par la CIPR. 
Pour le cancer du sein, les écarts sont considérables entre tranches d'âge et entre modèles (tableau V), même si le choix de l'âge de 25 ans amplifie l'effet ${ }^{7}$. II reste certain que le BEIR $V$ estime globalement un risque 4 à 5 fois plus faible que ne le fait I'UNSCEAR, probablement à cause de l'introduction des autres données. Ce facteur 4 à 5 semble, d'ailleurs, indiquer un très faible poids attribué aux données d'Hiroshima et Nagasaki. La présentation d'indicateurs du risque à la vie entière basés sur les populations différentes avait presque totalement occulté cet écart (tableau II). La comparaison des résultats sur une même population de référence est donc essentielle si l'on veut juger des impacts des techniques d'ajustement.

TABLEAU IV

Excès de cancers du poumon associès à une dose de $0,1 \mathrm{~Sv}$ Nombre de cas pour 100000 par sievert.

\begin{tabular}{|l|c|c|}
\hline \multicolumn{1}{|c|}{ Modèle } & Exposition à 25 ans & Exposition à 55 ans \\
\hline $\begin{array}{l}\text { Risque relatif constant } \\
\text { UNSCEAR }\end{array}$ & 172 & 146 \\
$\begin{array}{l}\text { Risque relatif variable } \\
\text { UNSCEAR } \\
\begin{array}{l}\text { Risque relatif variable } \\
\text { BEIR V }\end{array}\end{array}$ & 55 & 183 \\
\hline
\end{tabular}

TABLEAU $V$

Excès de cancers du sein associès à une dose de $0,1 \mathrm{SV}$ Nombre de cas pour 100000 par sievert.

\begin{tabular}{|l|c|c|}
\hline \multicolumn{1}{|c|}{ Modèle } & Exposition à 25 ans & Exposition à 55 ans \\
\hline $\begin{array}{l}\text { Risque relatif constant } \\
\text { UNSCEAR }\end{array}$ & 41 & 20 \\
$\begin{array}{l}\text { Risque relatif variable } \\
\text { UNSCEAR }\end{array}$ & 33 & 0,5 \\
$\begin{array}{l}\text { Risque relatif variable } \\
\text { BEIR V }\end{array}$ & 10 & 1,3 \\
\hline
\end{tabular}

\section{CONCLUSIONS}

Passant en revue les principales alternatives dans la modélisation qui aboutit aux calculs de risque à la vie entière, un certain nombre de faits marquants sont à noter.

- Les principaux facteurs de variabilité sont le choix de la population de référence, la base de données, et le modèle quil décrit l'évolution de l'excès de risque avec l'âge.

7. En effet, avant 25 ans, l'écart entre "BEIR V" et "risque relatif variable" de l'UNSCEAR aurait été plus faible, car pour le BEIR $V$ le risque est divisé par 6 entre 15 et 25 ans, alors qu'il n'est divisé que par 2 dans les coefficients utilisés par I'UNSCEAR). 
- L'effet de la distribution temporelle de la dose n'est pas discuté dans ce type d'analyse. II intervient - à tort ou à raison - comme un facteur multiplicatif s'appliquant au résultat global.

- Si l'on estime le risque sur une population globale, tous âges et tous cancers confondus, la plupart des effets se compensent et les écarts sont relativement faibles.

- Lorsque l'on recherche un coefficient pour un cancer spécifique, en utilisant le même coefficient de risque primaire, on peut observer un facteur 3 à 5 pour certains sites suivant les pays de référence. Ce même facteur se rencontre - semble-t-il - quand le coefficient primaire varie en fonction des données épidémiologiques. On note aussi l'importance, peu commentée, du "modèle de transport".

- La pratique de la CIPR, qui définit une population de référence implicite en moyennant sur les pays et sur les modèles de transport, permet d'aboutir à des indicateurs de risque "par cancer" stables mais qu'il serait hasardeux d'appliquer dans une optique d'"évaluation" ou d'optimisation. De toute évidence, le souci de garder un système simple et efficace a primé sur celui de la précision des prédictions.

- Lorsque l'on descend à un niveau encore plus fin en faisant jouer non seulement le type de cancer mais aussi l'effet de l'âge à l'exposition, l'instabilité devient très grande, qu'il s'agisse de l'effet âge dans un modèle donné ou de l'effet modèle à un âge donné. A ceci peut encore s'ajouter l'effet population de référence : pour le cancer du sein, entre l'application du modèle BEIR à une japonaise de 25 ans et de celui de I'UNSCEAR (risque relatif variable) à une anglaise de 15 ans, on obtient un facteur 35. L'écart dépasse un facteur 250 si le premier terme de la comparaison est l'application du modèle BEIR à une japonaise de 55 ans. Cette instabilité ne doit pas étonner puisqu'il faut répartir 3 à 400 cancers en excès entre une dizaine d'organes, et divers groupes d'âge tant à l'irradiation qu'à l'observation.

On peut en déduire qu'il est possible d'utiliser les relations dose-effets pour une irradiation globale, sous réserve, bien sûr, que l'on accepte l'idée que les études épidémiologiques sont une base valable. Pour un cancer donné, on ne peut atteindre que des ordres de grandeur - ce que certains jugent suffisants dans une optique de radioprotection. A ce sujet, il semble que toutes les discussions sur les modélisations épidémiologiques et biologiques de la cancérogenèse resteront illusoires tant que l'on ne disposera pas d'un "standard" pour les calculs du risque à la vie entière, normalisant les indicateurs, les modes de calcul et la population de référence.

Pour des cancers et des âges donnés, la variabilité est bien trop grande, en général, pour évaluer les risques, ce qui pose des problèmes si l'on veut utiliser des résultats du type de ceux de I'UNSCEAR, du BEIR ou de la CIPR pour décider, par exemple, de l'opportunité d'un dépistage. Cependant, si les écarts considérables cités ici ne sont pas, en général, réductibles, ils peuvent l'être sur des cas particuliers qu'il serait souhaitable d'identifier. Pour ces cas particuliers (par exemple cancer du sein pour des âges à l'irradiation de 10-20 ans), les stratégies d'estimation 
actuelles pourraient être modifiées de façon à être adaptées aux questions posées, notamment en donnant plus de poids aux données directement pertinentes.

\section{Annexe \\ Modèles statistiques utilisés par les comités}

Les données d'Hiroshima et de Nagasaki, même si elles ne sont pas seules utilisées, constituent la base de l'analyse. Elles ont été mises en forme par le RERF qui a constitué 8700 "cellules" à partir des groupements des 76000 individus suivis. Les groupements ont été faits selon les paramètres suivants :

PO : période d'observation : tranches de 5 ans entre 1950 et 1985

Al : âge à l'irradiation : $<10,10-19,20-29,30-39,40-49,>50$

$\mathrm{AO}$ : âge à l'observation $:<20,20-29,30-39,40-49,50-59,60-70,>70$

D : dose (Gy, Kerma) : 0, 0,01-0,05, 0,06-0,09;0,01-0,19;0,2-0,49;

$\mathrm{V}$ : ville : Hiroshima ; Nagasaki.

$S:$ : sexe $H$; F.

Dans chacune de ces cellules, on compte un nombre de personne-ans (PA) et de décès $M$. En notant j le groupe de dose et $s$ la "strate" (croisement de tous les autres facteurs : âge, ville, sexe...) on définit alors les risques relatifs (eq. 2) et absolus (eq.1) pour un groupe de dose, $M(s, 0)$ étant le nombre attendu de décès dans la strate si les taux étalent ceux du groupe témoin :

$$
\begin{array}{ll}
M(s, j)=R A(s, j)+M(s, 0) & \text { eq } 1 \\
M(s, j)=R R(s, j) \times M(s, 0) & \text { eq } 2
\end{array}
$$

Jusqu'à cette étape, il s'agit de la simple définition des paramètres usuels d'une étude épidémiologique et il n'y a pas à proprement parler de modélisation. La RERF, et à sa suite l'UNSCEAR et le BEIR ont choisi le groupe "dose 0" comme groupe témoin, ce qui est un premier choix, peu contesté. Notons que ceci n'est pas le cas pour des irradiations thérapeutiques, où il faut souvent prendre un groupe témoin externe. Ensuite, les approches de l'UNSCEAR et du BEIR diffèrent. Le BEIR V "estime" la mortalité dans le groupe témoin en ajustant les paramètres à une fonction explicite des âges à l'irradiation et à l'observation (cf. eq. 3) :

$M(s, 0)=P A(s, 0) \times \exp .\left(a_{0}+a_{1} \operatorname{Ln} A O+a_{2} \operatorname{Ln}{ }^{2} A O+a_{3} s+a_{4} \operatorname{Ln} A I+\ldots\right)$ eq 3

La RERF et l'UNSCEAR se contentent de mesurer le nombre observé de décès dans chaque strate ou dans des regroupements et font autant d'ajustements séparés.

Les deux comités ont ensuite décidé d'estimer alternativement les coefficients de risque relatif et de risque absolu en fonction de la dose. Le BEIR V avait, de plus, tenté l'utilisation d'un modèle "mixte" auquel il a renoncé. Les modèles retenus, ou favorisés, étant ceux du risque relatif, on décrit maintenant ce cas.

L'UNSCEAR s'est limité à une équation simple, où le risque relatif dépend uniquement de la dose et de façon linéaire :

$$
R R(s, j)=M(s, j) / M(s, 0)=1+b D(s, j) \quad \text { eq. } 4
$$

L'ajustement a ensuite été effectué soit en regroupant toutes les strates (risque relatif constant), soit à partir de regroupements partiels (risque relatif en fonction du sexe et de l'âge à l'irradiation). Ces chiffres fournissent directement 
les coefficients de risque primaire utilisés par I'UNSCEAR (rapport et annexe technique) et la CIPR. Ils sont obtenus par une technique similaire à celle du BEIR (estimation du maximum de vraisemblance en supposant que le nombre de décès suit une loi de Poisson). II faut noter que, si le coefficient primaire associé au risque relatif constant est la moyenne des coefficients de risque variables, son utilisation pour le calcul du risque à la vie entière ne donne pas le même résultat que la moyenne des résultats obtenus avec les coefficients spécifiques. Moyenner "avant" introduit un biais vers le bas qui explique que les résultats de l'annexe de I'UNSCEAR et de la CIPR solent supérieurs de $30 \%$ à ceux de l'estimation proposée par I'UNSCEAR.

Le BEIR $V$ propose un modèle plus complexe, où le risque relatif dépend de nombreux facteurs, ne retenant pour chaque cancer que les plus significatifs des coefficients :

$$
\begin{aligned}
& R R(s, j)=1+\left(b, D+b_{2} D^{2}\right) f(s) \\
& \text { avec } f(s)=\exp \left(c_{1} \operatorname{Ln} A O+c_{2} \operatorname{Ln} A I+c_{3} \operatorname{Ln}(A O-A I)+\right. \\
& \left.C_{4} L N((A O-A l) / 20)+C_{5} L^{2} A O+\ldots\right)
\end{aligned}
$$

Selon les cancers, les paramètres significatifs ne sont donc pas les mêmes. Si cette approche permet de bien reconstituer les données, la question de la stabilité des prédictions doit être posée.

La RERF a utilisé un modèle similaire non pas pour l'ajustement, mais pour tester les effets des différents paramètres (sexe, ville...).

\section{RÉFÉRENCES}

[1] COMMISSION INTERNATIONALE DE PROTECTION RADIOLOGIQUE (CIPR). Projet de recommandations, 1990.

[2] DARBY S., FAGNANI F., HUBERT Ph., SCHNEIDER T., THOMAS D., VARTH M., WEISS K. - Measures of lifetime detriment from radiation exposure : principles and methods (Rapport CEPN No 175). Paris : CEPN, 1990.

[3] ETATS-UNIS. National institute of health (NIH). - Report of the NIH ad hoc working group to develop radioepidemiological tables (NIH publication $\mathrm{N}^{\circ}$ 85-2748). Bethesda : NIH, 1988.

[4] ETATS-UNIS. Nuclear regulatory commission (NUREG). - Health effects models for nuclear power plant accident consequence analysis, Pt 2. Scientific bases for health effects models (NUREG/CR-4214 REV 1). Washington : NRC, 1989.

[5] HUBERT D. - Intérêt et limites de l'épidémiologie pour l'évaluation des risques de cancers radioinduits et l'établissement des normes de radioprotection. Radioprotection, 1990,25 (1) 19-41.

[6] HUBERT Ph. - Evaluation du risque à la vie entière dans les analyses de I'UNSCEAR, du BEIR et de la CIPR (Note SEGP-LSEES 90-08). Fontenay-auxRoses: CEA, 1990.

[7] INSTITUT NATIONAL DE LA SANTÉ ET DE LA RECHERCHE MÉDICALE (INSERM). - Statistiques de santé. Evolution de la mortalité par cancer en France entre 1950 et 1985. Paris : INSERM, 1989.

[8] Modèle des relations dose-effets aux faibles doses (Note CEPN). Fontenayaux-Roses : CEPN, 1987. 
[9] NATIONAL ACADEMY OF SCIENCES (NAS). - Health effects of exposure to low-levels of ionizing radiation (BEIR $V$ report). Washington DC : NAS, 1990.

[10] NATIONAL COUNCIL ON RADIATION PROTECTION AND MEASUREMENTS (NCRP). - Influence of dose and its distribution in time on dose-response relationships for low LET radiation (NCRP report $N^{0}$ 64). Bethesda : NCRP, 1980 .

[11] SHIMIZU Y., KATO H., SCHULL W.J. - Life span study report 11. Part 2. Cancer mortality in the years 1950-85 based on the recently revised doses (DS86) (RERF-TR 5-88). Hiroshima : Radiation effects research foundation, 1988.

[12] THOMAS D.C. - Models for predicting radiation risks in the BEIR V report. In : Low dose radiation : biological bases of risk assessment (BAVENSTOCK K., Ed.). New York : Plenum press, 1989.

[13] UNITED NATIONS SCIENTIFIC COMMITTEE ON THE EFFECTS OF ATOMIC RADIATION (UNSCEAR). - Sources, effects and risks of ionizing radiation. Annex F. Radiation carcinogenesis in man (E.88.IX.7). New York : Nations Unies, 1988. 